\title{
Stability of finite difference schemes for hyperbolic systems in two space dimensions
}

\author{
Jean-François Coulombel \\ CNRS \& Université Lille 1, Laboratoire Paul Painlevé, UMR CNRS 8524, \\ Cité scientifique, 59655 VILLENEUVE D'ASCQ Cedex, France \\ Email: jfcoulom@math.univ-lille1.fr
}

April 6, 2005

\begin{abstract}
We study the stability of some finite difference schemes for hyperbolic systems in two space dimensions. The grid is assumed to be cartesian, but the space steps in each direction are not necessarily equal. Our sufficient stability conditions are shown to be also necessary for one concrete example. We conclude with some numerical illustrations of our result.
\end{abstract}

AMS subject classification: 65M12, 65M06, 35L45.

Keywords: Hyperbolic systems, finite difference schemes, stability.

\section{Introduction}

Finite difference schemes are commonly used to approximate the solutions to hyperbolic systems of conservation laws. In this paper, we are interested in the stability of such finite difference schemes when applied to constant coefficients hyperbolic systems in two space dimensions. When applied to variable coefficients or nonlinear systems, the Courant-Friedrichs-Lewy condition that we derive can be seen as a local condition that needs to be satisfied in each cell of the grid.

We consider a symmetric hyperbolic system in two space dimensions:

$$
\begin{cases}\partial_{t} u+A_{1} \partial_{x_{1}} u+A_{2} \partial_{x_{2}} u=0, & t \geq 0, x \in \mathbb{R}^{2}, \\ u_{\left.\right|_{t=0}}=u_{0}, & x \in \mathbb{R}^{2} .\end{cases}
$$

The matrices $A_{1}$, and $A_{2}$ belong to $M_{d}(\mathbb{R})$, and are symmetric, so that the Cauchy problem (1) is well-posed in $L^{2}\left(\mathbb{R}^{2}\right)$, see e.g. [3]. Moreover, the solution of (1) satisfies

$$
\forall t \geq 0, \quad\|u(t)\|_{L^{2}\left(\mathbb{R}^{2}\right)}=\left\|u_{0}\right\|_{L^{2}\left(\mathbb{R}^{2}\right)} .
$$

We introduce a finite difference approximation of (1). Let $\Delta x_{1}$, and $\Delta x_{2}$ denote some space steps in the $x_{1}$, and $x_{2}$ directions, and let $\Delta t$ denote the time step. Then the vector $u_{j, k}^{n}$, where $(n, j, k) \in \mathbb{N} \times \mathbb{Z} \times \mathbb{Z}$, denotes an approximation of $u\left(n \Delta t, j \Delta x_{1}, k \Delta x_{2}\right)$. Following [1], we define

$$
\lambda_{1}:=\frac{\Delta t}{\Delta x_{1}}, \quad \lambda_{2}:=\frac{\Delta t}{\Delta x_{2}} .
$$

We refer to [1, chapter IV.3], and [2, chapter 6] for a general description of finite difference schemes for two-dimensional hyperbolic systems, and we shall thus assume that the reader is familiar with the basic $L^{2}$ stability theory of finite difference schemes (see e.g. [1, page 348]). In this paper, we shall study the stability of four finite difference schemes: 
- The two-dimensional Lax-Friedrichs scheme:

$$
u_{j, k}^{n+1}=\frac{1}{4}\left(u_{j-1, k}^{n}+u_{j+1, k}^{n}+u_{j, k-1}^{n}+u_{j, k+1}^{n}\right)-\frac{\lambda_{1}}{2} A_{1}\left(u_{j+1, k}^{n}-u_{j-1, k}^{n}\right)-\frac{\lambda_{2}}{2} A_{2}\left(u_{j, k+1}^{n}-u_{j, k-1}^{n}\right) .
$$

- The dimensional-splitting Lax-Friedrichs scheme:

$$
\begin{aligned}
& u_{j, k}^{n+1 / 2}=\frac{1}{2}\left(u_{j-1, k}^{n}+u_{j+1, k}^{n}\right)-\frac{\lambda_{1}}{2} A_{1}\left(u_{j+1, k}^{n}-u_{j-1, k}^{n}\right), \\
& u_{j, k}^{n+1}=\frac{1}{2}\left(u_{j, k-1}^{n+1 / 2}+u_{j, k+1}^{n+1 / 2}\right)-\frac{\lambda_{2}}{2} A_{2}\left(u_{j, k+1}^{n+1 / 2}-u_{j, k-1}^{n+1 / 2}\right) .
\end{aligned}
$$

- The two-dimensional Godunov scheme:

$$
\begin{aligned}
u_{j, k}^{n+1}=u_{j, k}^{n}-\frac{\lambda_{1}}{2} A_{1} & \left(u_{j+1, k}^{n}-u_{j-1, k}^{n}\right)-\frac{\lambda_{1}}{2}\left|A_{1}\right|\left(2 u_{j, k}^{n}-u_{j+1, k}^{n}-u_{j-1, k}^{n}\right) \\
& \quad-\frac{\lambda_{2}}{2} A_{2}\left(u_{j, k+1}^{n}-u_{j, k-1}^{n}\right)-\frac{\lambda_{2}}{2}\left|A_{2}\right|\left(2 u_{j, k}^{n}-u_{j, k+1}^{n}-u_{j, k-1}^{n}\right) .
\end{aligned}
$$

- The dimensional-splitting Godunov scheme:

$$
\begin{array}{r}
u_{j, k}^{n+1 / 2}=u_{j, k}^{n}-\frac{\lambda_{1}}{2} A_{1}\left(u_{j+1, k}^{n}-u_{j-1, k}^{n}\right)-\frac{\lambda_{1}}{2}\left|A_{1}\right|\left(2 u_{j, k}^{n}-u_{j+1, k}^{n}-u_{j-1, k}^{n}\right), \\
u_{j, k}^{n+1}=u_{j, k}^{n+1 / 2}-\frac{\lambda_{2}}{2} A_{2}\left(u_{j, k+1}^{n+1 / 2}-u_{j, k-1}^{n+1 / 2}\right)-\frac{\lambda_{2}}{2}\left|A_{2}\right|\left(2 u_{j, k}^{n+1 / 2}-u_{j, k+1}^{n+1 / 2}-u_{j, k-1}^{n+1 / 2}\right) .
\end{array}
$$

We do not know whether the terminology is really standard, but we hope that it is clear enough. Recall that in (5), and (6), the matrices $\left|A_{1,2}\right|$ are defined as follows: let $P_{1,2}$ denote orthogonal matrices that diagonalize $A_{1,2}$ :

$$
P_{1}^{-1} A_{1} P_{1}=\operatorname{diag}\left(\alpha_{1}, \ldots, \alpha_{d}\right), \quad P_{2}^{-1} A_{2} P_{2}=\operatorname{diag}\left(\beta_{1}, \ldots, \beta_{d}\right) .
$$

Then the matrices $\left|A_{1}\right|$, and $\left|A_{2}\right|$, are given by:

$$
P_{1}^{-1}\left|A_{1}\right| P_{1}=\operatorname{diag}\left(\left|\alpha_{1}\right|, \ldots,\left|\alpha_{d}\right|\right), \quad P_{2}^{-1}\left|A_{2}\right| P_{2}=\operatorname{diag}\left(\left|\beta_{1}\right|, \ldots,\left|\beta_{d}\right|\right) .
$$

Observe that $\left|A_{1}\right|$, and $\left|A_{2}\right|$ are symmetric, nonnegative matrices. They are positive definite if $A_{1}$, and $A_{2}$ are nonsingular.

When $\lambda_{1}=\lambda_{2}$, the stability of (3) was completely analyzed in [4], even in the case of variable coefficients. The extension to different space steps is easy, but we give it here to enlight the difference between the stability criteria for (3) and (5).

In all what follows, the spectral radius of a square matrix $M$ with complex entries is denoted $\rho(M)$. Our main result is the following:

Theorem 1. - The scheme (3) is stable in $\ell^{2}\left(\mathbb{Z}^{2}\right)$ if

$$
\forall \vartheta \in[0,2 \pi], \quad \rho\left(\lambda_{1} \cos \vartheta A_{1}+\lambda_{2} \sin \vartheta A_{2}\right) \leq \frac{1}{\sqrt{2}} .
$$

- The scheme (4) is stable in $\ell^{2}\left(\mathbb{Z}^{2}\right)$ if, and only if

$$
\max \left(\lambda_{1} \rho\left(A_{1}\right), \lambda_{2} \rho\left(A_{2}\right)\right) \leq 1 .
$$


- The scheme $(5)$ is stable in $\ell^{2}\left(\mathbb{Z}^{2}\right)$ if

$$
\lambda_{1} \rho\left(A_{1}\right)+\lambda_{2} \rho\left(A_{2}\right) \leq 1 .
$$

If $A_{1}$, and $A_{2}$ are nonsingular, and if $\lambda_{1} \rho\left(A_{1}\right)+\lambda_{2} \rho\left(A_{2}\right)<1$, then the scheme (5) is dissipative (in Kreiss' sense) of order 2 . Namely, if $\mathbb{G}\left(\xi_{1}, \xi_{2}\right)$ denotes the symbol of the scheme (5), there exists a constant $c>0$ such that

$\forall\left(\xi_{1}, \xi_{2}\right) \in\left[-\frac{\pi}{\Delta x_{1}}, \frac{\pi}{\Delta x_{1}}\right] \times\left[-\frac{\pi}{\Delta x_{2}}, \frac{\pi}{\Delta x_{2}}\right], \quad \rho\left(\mathbb{G}\left(\xi_{1}, \xi_{2}\right)\right) \leq 1-c\left(\left(\xi_{1} \Delta x_{1}\right)^{2}+\left(\xi_{2} \Delta x_{2}\right)^{2}\right)$.

- The scheme (6) is stable in $\ell^{2}\left(\mathbb{Z}^{2}\right)$ if, and only if

$$
\max \left(\lambda_{1} \rho\left(A_{1}\right), \lambda_{2} \rho\left(A_{2}\right)\right) \leq 1 .
$$

For the schemes (3), and (5), Theorem 1 only gives sufficient stability conditions. For a particular system, one may hope to get less restrictive stability conditions. However, the following result shows that the conditions of Theorem 1 are optimal in the general case (that is, they can not be improved for all symmetric hyperbolic systems):

Theorem 2. Let $A_{1}$, and $A_{2}$ be given by

$$
A_{1}=\left(\begin{array}{ll}
0 & 1 \\
1 & 0
\end{array}\right), \quad A_{2}=\left(\begin{array}{cc}
-1 & 0 \\
0 & 1
\end{array}\right) .
$$

Then we have the following necessary and sufficient conditions:

- The scheme (3) is stable in $\ell^{2}\left(\mathbb{Z}^{2}\right)$ if, and only if $\sqrt{2} \max \left(\lambda_{1}, \lambda_{2}\right) \leq 1$, which is equivalent to (9).

- The scheme (5) is stable in $\ell^{2}\left(\mathbb{Z}^{2}\right)$ if, and only if $\lambda_{1}+\lambda_{2} \leq 1$.

The paper is organized as follows. In section 2, we prove the first two items of Theorem 1 , and we also give a Lax-Friedrichs type scheme that is always unstable. We give this example in order to highlight the fact that one should be cautious when constructing two-dimensional schemes by simply adding one-dimensional schemes in each direction. Such an operation may yield instabilities. In section 3 , we prove the last two items of Theorem 1 . Then in section 4 , we prove Theorem 2. Eventually, in section 5, we compare the dissipativity of the Lax-Friedrichs and Godunov schemes with the help of numerical simulations. We shall also discuss the choice of the space steps.

\section{Stability of Lax-Friedrichs type schemes}

\subsection{An unstable Lax-Friedrichs type scheme}

There are many possible ways to construct a finite difference schemes in two space dimensions. As a first guess, one could think that it is enough to add the one-dimensional Lax-Friedrichs fluxes in each direction. Such a procedure yields the following scheme (see e.g. [1, page 346]):

$u_{j, k}^{n+1}=\frac{1}{2}\left(u_{j-1, k}^{n}+u_{j+1, k}^{n}+u_{j, k-1}^{n}+u_{j, k+1}^{n}-2 u_{j, k}^{n}\right)-\frac{\lambda_{1}}{2} A_{1}\left(u_{j+1, k}^{n}-u_{j-1, k}^{n}\right)-\frac{\lambda_{2}}{2} A_{2}\left(u_{j, k+1}^{n}-u_{j, k-1}^{n}\right)$.

The symbol $G$ of this scheme is computed by using a Fourier transform in the space variables. We obtain:

$$
G\left(\xi_{1}, \xi_{2}\right)=\left(\cos \left(\xi_{1} \Delta x_{1}\right)+\cos \left(\xi_{2} \Delta x_{2}\right)-1\right) I_{d}-i\left(\lambda_{1} \sin \left(\xi_{1} \Delta x_{1}\right) A_{1}+\lambda_{2} \sin \left(\xi_{2} \Delta x_{2}\right) A_{2}\right) .
$$

In particular, when $\xi_{1} \Delta x_{1}=\xi_{2} \Delta x_{2}=\pi$, the symbol $G$ equals $-3 I_{d}$, and the scheme is unstable in $\ell^{2}\left(\mathbb{Z}^{2}\right)$. 


\subsection{Stability of Lax-Friedrichs scheme}

We now study the scheme (3). Its symbol is computed by applying a Fourier transform in the space variables. We get

$$
G^{L F}\left(\xi_{1}, \xi_{2}\right)=\frac{1}{2}\left(\cos \left(\xi_{1} \Delta x_{1}\right)+\cos \left(\xi_{2} \Delta x_{2}\right)\right) I_{d}-i\left(\lambda_{1} \sin \left(\xi_{1} \Delta x_{1}\right) A_{1}+\lambda_{2} \sin \left(\xi_{2} \Delta x_{2}\right) A_{2}\right) .
$$

The matrices $A_{1,2}$ are symmetric. Therefore, the matrix $G^{L F}\left(\xi_{1}, \xi_{2}\right)$ is normal for all $\left(\xi_{1}, \xi_{2}\right)$.

The scheme (3) is thus stable if, and only if:

$$
\forall\left(\xi_{1}, \xi_{2}\right) \in \mathbb{R}^{2}, \quad I_{d}-G^{L F}\left(\xi_{1}, \xi_{2}\right)^{*} G^{L F}\left(\xi_{1}, \xi_{2}\right) \geq 0 .
$$

To simplify the computations, we denote $\zeta_{k}=\xi_{k} \Delta x_{k}, k=1,2$. Following [4], we compute

$$
\begin{aligned}
I_{d}-G^{L F}\left(\xi_{1}, \xi_{2}\right)^{*} G^{L F}\left(\xi_{1}, \xi_{2}\right)=\left(\frac{1}{2}\left(\sin ^{2} \zeta_{1}+\sin ^{2} \zeta_{2}\right)+\frac{1}{4}\left(\cos \zeta_{1}-\cos \zeta_{2}\right)^{2}\right) I_{d} & \\
& -\left(\lambda_{1} \sin \zeta_{1} A_{1}+\lambda_{2} \sin \zeta_{2} A_{2}\right)^{2} .
\end{aligned}
$$

Choosing $\vartheta$ such that

$$
\sin \zeta_{1}=\cos \vartheta \sqrt{\sin ^{2} \zeta_{1}+\sin ^{2} \zeta_{2}}, \quad \sin \zeta_{2}=\sin \vartheta \sqrt{\sin ^{2} \zeta_{1}+\sin ^{2} \zeta_{2}},
$$

we end up with

$$
I_{d}-G^{L F}\left(\xi_{1}, \xi_{2}\right)^{*} G^{L F}\left(\xi_{1}, \xi_{2}\right) \geq\left(\sin ^{2} \zeta_{1}+\sin ^{2} \zeta_{2}\right)\left(\frac{1}{2} I_{d}-\left(\lambda_{1} \cos \vartheta A_{1}+\lambda_{2} \sin \vartheta A_{2}\right)^{2}\right) .
$$

The first item of Theorem 1 follows, by recalling that for a hermitian matrix $H$ (and more generally for a normal matrix), the hermitian norm of $H$ (that is, the norm induced by the hermitian norm in $\mathbb{C}^{d}$ ) equals the spectral radius $\rho(H)$.

\subsection{Stability of the dimensional-splitting Lax-Friedrichs scheme}

We now study the scheme (4). Its symbol is given by

$$
G^{L F s}\left(\xi_{1}, \xi_{2}\right)=\left[\cos \left(\xi_{2} \Delta x_{2}\right) I_{d}-i \lambda_{2} \sin \left(\xi_{2} \Delta x_{2}\right) A_{2}\right]\left[\cos \left(\xi_{1} \Delta x_{1}\right) I_{d}-i \lambda_{1} \sin \left(\xi_{1} \Delta x_{1}\right) A_{1}\right] .
$$

Choosing either $\xi_{1}=0$, or $\xi_{2}=0$, it is clear that the stability of (4) implies the stability of each corresponding one-dimensional Lax-Friedrichs schemes. Therefore, if (4) is stable, then $\lambda_{1} \rho\left(A_{1}\right)$, and $\lambda_{2} \rho\left(A_{2}\right)$ are both less than 1 .

Assume now that both $\lambda_{1} \rho\left(A_{1}\right)$, and $\lambda_{2} \rho\left(A_{2}\right)$ are less than 1. From (14), we see that the symbol $G^{L F s}\left(\xi_{1}, \xi_{2}\right)$ is the product of two normal matrices, each of which has a spectral radius bounded by 1 . For a normal matrix, the spectral radius coincides with the hermitian norm, which implies that the hermitian norm of $G^{L F s}\left(\xi_{1}, \xi_{2}\right)$ is less than 1. This ensures that (4) is stable.

\section{Stability of Godunov type schemes}

\subsection{Stability of the two-dimensional Godunov scheme}

The symbol of the Godunov scheme (5) is

$$
\begin{aligned}
\mathbb{G}\left(\xi_{1}, \xi_{2}\right)=I_{d}-2\left(\lambda_{1} \sin ^{2}\left(\frac{\xi_{1} \Delta x_{1}}{2}\right)\left|A_{1}\right|+\right. & \left.\lambda_{2} \sin ^{2}\left(\frac{\xi_{2} \Delta x_{2}}{2}\right)\left|A_{2}\right|\right) \\
& -i\left(\lambda_{1} \sin \left(\xi_{1} \Delta x_{1}\right) A_{1}+\lambda_{2} \sin \left(\xi_{2} \Delta x_{2}\right) A_{2}\right) .
\end{aligned}
$$


In general, the matrix $\mathbb{G}\left(\xi_{1}, \xi_{2}\right)$ is not normal for all values of $\left(\xi_{1}, \xi_{2}\right)$. As a matter of fact, the reader can check that $\mathbb{G}\left(\xi_{1}, \xi_{2}\right)$ is normal if, and only if the matrices $A_{1}$, and $A_{2}$ satisfy

$$
A_{1}\left|A_{2}\right|-\left|A_{2}\right| A_{1}=A_{2}\left|A_{1}\right|-\left|A_{1}\right| A_{2}=0 .
$$

We shall not assume that these conditions are satisfied. Instead, we are going to show that under the condition (11), one has

$$
\forall z \in \mathbb{C}, \quad|z|>1, \quad\left\|\left(\mathbb{G}\left(\xi_{1}, \xi_{2}\right)-z I_{d}\right)^{-1}\right\| \leq \frac{1}{|z|-1},
$$

where $\|\cdot\|$ denotes the usual hermitian norm in $\mathbb{C}^{d}$, as well as the induced matrix norm.

From the well-known Kreiss' matrix Theorem, see e.g. [2, Theorem 5.2.4], the inequality (16) yields the stability of the difference scheme (5).

Assume first of all that, under the condition (11), we can prove the inequality

$$
\forall X \in \mathbb{C}^{d}, \quad\left|X^{*} \mathbb{G}\left(\xi_{1}, \xi_{2}\right) X\right| \leq\|X\|^{2} .
$$

In particular, the spectral radius $\rho\left(\mathbb{G}\left(\xi_{1}, \xi_{2}\right)\right)$ is less than 1 . Furthermore, let $z \in \mathbb{C}$ with $|z|>1$, let $Y \in \mathbb{C}^{d}$, and let $X \in \mathbb{C}^{d}$ be the unique solution to

$$
\left(\mathbb{G}\left(\xi_{1}, \xi_{2}\right)-z I_{d}\right) X=Y .
$$

We get

$$
z\|X\|^{2}=X^{*} \mathbb{G}\left(\xi_{1}, \xi_{2}\right) X-X^{*} Y .
$$

Using (17), and the Cauchy-Schwarz' inequality, (18) yields

$$
(|z|-1)\|X\|^{2} \leq\|X\|\|Y\|,
$$

from which we obtain (16). We thus only need to prove (17).

It is convenient to define $\eta_{k}=\xi_{k} \Delta x_{k} / 2, k=1,2$. Then the symbol $\mathbb{G}\left(\xi_{1}, \xi_{2}\right)$ reads

$$
\mathbb{G}\left(\xi_{1}, \xi_{2}\right)=I_{d}-2\left(\lambda_{1} \sin ^{2} \eta_{1}\left|A_{1}\right|+\lambda_{2} \sin ^{2} \eta_{2}\left|A_{2}\right|\right)-i\left(\lambda_{1} \sin \left(2 \eta_{1}\right) A_{1}+\lambda_{2} \sin \left(2 \eta_{2}\right) A_{2}\right) .
$$

Let $X \in \mathbb{C}^{d}$, with $\|X\|=1$. Using the symmetry of $A_{1},\left|A_{1}\right|, A_{2}$, and $\left|A_{2}\right|$, we compute:

$$
\begin{aligned}
\left|X^{*} \mathbb{G}\left(\xi_{1}, \xi_{2}\right) X\right|^{2}=\left[1-2\left(\lambda_{1} \sin ^{2} \eta_{1} X^{*}\right.\right. & \left.\left.\left|A_{1}\right| X+\lambda_{2} \sin ^{2} \eta_{2} X^{*}\left|A_{2}\right| X\right)\right]^{2} \\
& +\left[\lambda_{1} \sin \left(2 \eta_{1}\right) X^{*} A_{1} X+\lambda_{2} \sin \left(2 \eta_{2}\right) X^{*} A_{2} X\right]^{2} .
\end{aligned}
$$

Observing that

$\left|\lambda_{1} \sin \left(2 \eta_{1}\right) X^{*} A_{1} X+\lambda_{2} \sin \left(2 \eta_{2}\right) X^{*} A_{2} X\right| \leq \lambda_{1}\left|\sin \left(2 \eta_{1}\right)\right| X^{*}\left|A_{1}\right| X+\lambda_{2}\left|\sin \left(2 \eta_{2}\right)\right| X^{*}\left|A_{2}\right| X$, we can expand (19), and derive the inequality:

$$
\begin{aligned}
\left|X^{*} \mathbb{G}\left(\xi_{1}, \xi_{2}\right) X\right|^{2} \leq 1-4\left(\mu_{1} \sin ^{2} \eta_{1}+\mu_{2} \sin ^{2} \eta_{2}\right)+4\left(\mu_{1}\right. & \left.\sin ^{2} \eta_{1}+\mu_{2} \sin ^{2} \eta_{2}\right)^{2} \\
& +\left(\mu_{1}\left|\sin 2 \eta_{1}\right|+\mu_{2} \sin \left|2 \eta_{2}\right|\right)^{2}
\end{aligned}
$$

where we have set

$$
\mu_{k}:=\lambda_{k} X^{*}\left|A_{k}\right| X, \quad k=1,2 .
$$

Expanding the right-hand side of (20), we obtain

$$
\begin{aligned}
\left|X^{*} \mathbb{G}\left(\xi_{1}, \xi_{2}\right) X\right|^{2} \leq 1+4\left(\mu_{1}^{2}-\mu_{1}\right) \sin ^{2} \eta_{1} & +4\left(\mu_{2}^{2}-\mu_{2}\right) \sin ^{2} \eta_{2} \\
& +2 \mu_{1} \mu_{2}\left(4 \sin ^{2} \eta_{1} \sin ^{2} \eta_{2}+\left|\sin 2 \eta_{1}\right|\left|\sin 2 \eta_{2}\right|\right) .
\end{aligned}
$$

To complete the proof, we shall use the following Lemma: 
Lemma 1. Let $\left(\eta_{1}, \eta_{2}\right) \in \mathbb{R}^{2}$, and let $\mathcal{T}$ denote the triangle:

$$
\mathcal{T}:=\left\{\left(y_{1}, y_{2}\right) \in \mathbb{R}^{2} / y_{1} \geq 0, y_{2} \geq 0, y_{1}+y_{2} \leq 1\right\} .
$$

Then for all $\left(y_{1}, y_{2}\right) \in \mathcal{T}$, one has

$$
\left(y_{1}^{2}-y_{1}\right) \sin ^{2} \eta_{1}+\left(y_{2}^{2}-y_{2}\right) \sin ^{2} \eta_{2}+2 y_{1} y_{2}\left(\sin ^{2} \eta_{1} \sin ^{2} \eta_{2}+\frac{1}{4}\left|\sin 2 \eta_{1}\right|\left|\sin 2 \eta_{2}\right|\right) \leq 0 .
$$

From the definition (21), we have $\mu_{1} \geq 0$, and $\mu_{2} \geq 0$. The inequality $\mu_{1}+\mu_{2} \leq 1$ follows from the condition (11). Then using Lemma 1 in (22), we obtain (17). (Note that it is sufficient to prove (17) on the unit sphere by homogeneity). We now prove Lemma 1 . Define

$$
g\left(y_{1}, y_{2}\right):=\left(y_{1}^{2}-y_{1}\right) \sin ^{2} \eta_{1}+\left(y_{2}^{2}-y_{2}\right) \sin ^{2} \eta_{2}+2 y_{1} y_{2}\left(\sin ^{2} \eta_{1} \sin ^{2} \eta_{2}+\frac{1}{4}\left|\sin 2 \eta_{1}\right|\left|\sin 2 \eta_{2}\right|\right)
$$

Using the inequality

$$
\begin{array}{r}
\sin ^{2} \eta_{1} \sin ^{2} \eta_{2}+\frac{1}{4}\left|\sin 2 \eta_{1}\right|\left|\sin 2 \eta_{2}\right|=\left|\sin \eta_{1}\right|\left|\sin \eta_{2}\right|\left(\left|\sin \eta_{1}\right|\left|\sin \eta_{2}\right|+\left|\cos \eta_{1}\right|\left|\cos \eta_{2}\right|\right) \\
\leq\left|\sin \eta_{1}\right|\left|\sin \eta_{2}\right|
\end{array}
$$

one easily checks that $g$ is a convex function. Therefore, the maximum of $g$ on the triangle $\mathcal{T}$ is attained on the edges of the triangle. We compute $g$ on each edge of $\mathcal{T}$ :

$$
\begin{aligned}
& g\left(y_{1}, 0\right)=\left(y_{1}^{2}-y_{1}\right) \sin ^{2} \eta_{1} \leq 0, \\
& g\left(0, y_{2}\right)=\left(y_{2}^{2}-y_{2}\right) \sin ^{2} \eta_{2} \leq 0, \\
& g\left(y_{1}, 1-y_{1}\right)=\left(y_{1}^{2}-y_{1}\right)\left[\sin ^{2} \eta_{1}+\sin ^{2} \eta_{2}-2\left(\sin ^{2} \eta_{1} \sin ^{2} \eta_{2}+\frac{1}{4}\left|\sin 2 \eta_{1}\right|\left|\sin 2 \eta_{2}\right|\right)\right] \leq 0 .
\end{aligned}
$$

Consequently, $g$ is nonpositive on $\mathcal{T}$, and Lemma 1 is proved.

\subsection{Dissipativity of the Godunov scheme}

We now assume that the matrices $A_{1}$, and $A_{2}$ are nonsingular, and that $\lambda_{1} \rho\left(A_{1}\right)+\lambda_{2} \rho\left(A_{2}\right)<1$. Consequently, there exists a constant $\delta>0$ such that for all $X \in \mathbb{C}^{d}$, with $\|X\|=1$, one has

$$
\mu_{1}:=\lambda_{1} X^{*}\left|A_{1}\right| X \geq \delta, \quad \mu_{2}:=\lambda_{2} X^{*}\left|A_{2}\right| X \geq \delta, \quad \mu_{1}+\mu_{2} \leq 1-\delta .
$$

For such a positive constant $\delta$, we define the triangle:

$$
\mathcal{T}_{\delta}:=\left\{\left(y_{1}, y_{2}\right) \in \mathbb{R}^{2} / y_{1} \geq \delta, y_{2} \geq \delta, y_{1}+y_{2} \leq 1-\delta\right\} .
$$

In order to show the dissipativity of the scheme (5), we use the inequality:

$$
\rho\left(\mathbb{G}\left(\xi_{1}, \xi_{2}\right)\right)^{2} \leq \max _{\|X\|=1}\left|X^{*} \mathbb{G}\left(\xi_{1}, \xi_{2}\right) X\right|^{2} \leq 1+4 \max _{\left(y_{1}, y_{2}\right) \in \mathcal{T}_{\delta}} g\left(y_{1}, y_{2}\right),
$$

see $(22)$, and (23). It is therefore sufficient to derive an upper bound of the function $g$ on the triangle $\mathcal{T}_{\delta}$. We have the following result:

Proposition 1. Let $\delta>0$ be fixed as above. Then for all $\left(\eta_{1}, \eta_{2}\right) \in[-\pi / 2, \pi / 2]^{2}$, one has

$$
\max _{\left(y_{1}, y_{2}\right) \in \mathcal{T}_{\delta}} g\left(y_{1}, y_{2}\right) \leq 0
$$

and the maximum is zero if, and only if $\left(\eta_{1}, \eta_{2}\right)=(0,0)$. Moreover, there exists a positive constant $c$ such that for all $\left(\eta_{1}, \eta_{2}\right) \in[-\pi / 2, \pi / 2]^{2}$, one has

$$
\max _{\left(y_{1}, y_{2}\right) \in \mathcal{T}_{\delta}} g\left(y_{1}, y_{2}\right) \leq-c\left(\eta_{1}^{2}+\eta_{2}^{2}\right) \text {. }
$$


Proof. From the definition (23) of the function $g$, it is clear that we only need to prove the result when $\left(\eta_{1}, \eta_{2}\right) \in[0, \pi / 2]^{2}$, which we assume from now on. Moreover, we already know that $g$ is convex, so it is sufficient to estimate the maximum of $g$ on the edges of the triangle $\mathcal{T}_{\delta}$.

When $y_{2} \in[\delta, 1-2 \delta]$, one has

$$
\begin{aligned}
g\left(\delta, y_{2}\right) & =\left(\delta^{2}-\delta\right) \sin ^{2} \eta_{1}+\left(y_{2}^{2}-y_{2}\right) \sin ^{2} \eta_{2}+2 \delta y_{2}\left(\sin ^{2} \eta_{1} \sin ^{2} \eta_{2}+\frac{1}{4} \sin 2 \eta_{1} \sin 2 \eta_{2}\right) \\
& \leq\left(\delta^{2}-\delta\right)\left(\sin ^{2} \eta_{1}+\sin ^{2} \eta_{2}\right)+2 \delta(1-2 \delta)\left(\sin ^{2} \eta_{1} \sin ^{2} \eta_{2}+\frac{1}{4} \sin 2 \eta_{1} \sin 2 \eta_{2}\right) \\
& \leq\left(\delta^{2}-\delta\right)\left(\sin \eta_{1}-\sin \eta_{2}\right)^{2}-2 \delta^{2}\left(\sin ^{2} \eta_{1} \sin ^{2} \eta_{2}+\frac{1}{4} \sin 2 \eta_{1} \sin 2 \eta_{2}\right) \leq 0 .
\end{aligned}
$$

In a completely similar, way, for $y_{1} \in[\delta, 1-2 \delta]$, we obtain

$$
g\left(y_{1}, \delta\right) \leq\left(\delta^{2}-\delta\right)\left(\sin \eta_{1}-\sin \eta_{2}\right)^{2}-2 \delta^{2}\left(\sin ^{2} \eta_{1} \sin ^{2} \eta_{2}+\frac{1}{4} \sin 2 \eta_{1} \sin 2 \eta_{2}\right) \leq 0 .
$$

Eventually, for $y_{1} \in[\delta, 1-2 \delta]$, we compute

$$
\begin{aligned}
g\left(y_{1}, 1-\delta-y_{1}\right) & =-y_{1}\left(1-\delta-y_{1}\right)\left[\sin ^{2} \eta_{1}+\sin ^{2} \eta_{2}-2\left(\sin ^{2} \eta_{1} \sin ^{2} \eta_{2}+\frac{1}{4} \sin 2 \eta_{1} \sin 2 \eta_{2}\right)\right] \\
& -\delta y_{1} \sin ^{2} \eta_{1}-\delta\left(1-\delta-y_{1}\right) \sin ^{2} \eta_{2} \\
& \leq\left(2 \delta^{2}-\delta\right)\left(\sin \eta_{1}-\sin \eta_{2}\right)^{2}-\delta^{2}\left(\sin ^{2} \eta_{1}+\sin ^{2} \eta_{2}\right) \\
& \leq-\delta^{2}\left(\sin ^{2} \eta_{1}+\sin ^{2} \eta_{2}\right) \leq-\frac{4 \delta^{2}}{\pi^{2}}\left(\eta_{1}^{2}+\eta_{2}^{2}\right) .
\end{aligned}
$$

Consequently, the maximum of $g$ on $\mathcal{T}_{\delta}$ is nonpositive, and the maximum is zero if, and only if $\eta_{1}=\eta_{2}=0$.

When $\left(\eta_{1}, \eta_{2}\right) \in[0, \pi / 4]^{2}$, one has

$$
\begin{aligned}
g\left(\delta, y_{2}\right) \leq 4\left(\delta^{2}-\delta\right) \sin ^{2} \frac{\eta_{1}-\eta_{2}}{2} \cos ^{2} \frac{\eta_{1}+\eta_{2}}{2} & -2 \delta^{2} \sin \eta_{1} \sin \eta_{2} \cos \left(\eta_{1}-\eta_{2}\right) \\
\leq & \leq-c(\delta)\left(\left(\eta_{1}-\eta_{2}\right)^{2}+\eta_{1} \eta_{2}\right) \leq-c(\delta)\left(\eta_{1}^{2}+\eta_{2}^{2}\right),
\end{aligned}
$$

and similarly

$$
g\left(y_{1}, \delta\right) \leq-c(\delta)\left(\eta_{1}^{2}+\eta_{2}^{2}\right) .
$$

We have thus obtained $(24)$ when $\left(\eta_{1}, \eta_{2}\right) \in[0, \pi / 4]^{2}$. When $\left(\eta_{1}, \eta_{2}\right) \in[0, \pi / 2]^{2} \backslash[0, \pi / 4]^{2}$, we have

$$
\max _{\left(y_{1}, y_{2}\right) \in \mathcal{T}_{\delta}} g\left(y_{1}, y_{2}\right) \leq-c(\delta) \leq-c(\delta)\left(\eta_{1}^{2}+\eta_{2}^{2}\right)
$$

so the proof of (24) is complete.

Using (24), we thus obtain:

$$
c\left(\eta_{1}^{2}+\eta_{2}^{2}\right) \leq 1-\rho\left(\mathbb{G}\left(\xi_{1}, \xi_{2}\right)\right)^{2} \leq 2\left[1-\rho\left(\mathbb{G}\left(\xi_{1}, \xi_{2}\right)\right)\right],
$$

so the scheme (5) is dissipative (in Kreiss' sense) of order 2. 


\subsection{Stability of the dimensional-splitting Godunov scheme}

The symbol of the scheme (6) is given by:

$$
\begin{aligned}
\mathbb{G}^{s}\left(\xi_{1}, \xi_{2}\right)=\left(I_{d}-2 \lambda_{2} \sin ^{2}\left(\frac{\xi_{2} \Delta x_{2}}{2}\right)\left|A_{2}\right|\right. & \left.-i \lambda_{2} \sin \left(\xi_{2} \Delta x_{2}\right) A_{2}\right) \\
& \left(I_{d}-2 \lambda_{1} \sin ^{2}\left(\frac{\xi_{1} \Delta x_{1}}{2}\right)\left|A_{1}\right|-i \lambda_{1} \sin \left(\xi_{1} \Delta x_{1}\right) A_{1}\right) .
\end{aligned}
$$

Observe that $A_{1}$, and $\left|A_{1}\right|$ commute, as well as $A_{2}$, and $\left|A_{2}\right|$. Therefore, $\mathbb{G}^{s}\left(\xi_{1}, \xi_{2}\right)$ is the product of two normal matrices. Then the proof of the last item of Theorem 1 follows exactly the arguments that we have used to study the dimensional-splitting Lax-Friedrichs scheme.

\section{Necessary stability conditions for a particular system}

In all this section, we study the finite difference schemes (3), and (5) when the matrices $A_{1}$, and $A_{2}$ are given by:

$$
A_{1}=\left(\begin{array}{ll}
0 & 1 \\
1 & 0
\end{array}\right), \quad A_{2}=\left(\begin{array}{cc}
-1 & 0 \\
0 & 1
\end{array}\right) .
$$

Observe that for all $\left(\xi_{1}, \xi_{2}\right) \in \mathbb{R}^{2}$, the eigenvalues of the matrix $\xi_{1} A_{1}+\xi_{2} A_{2}$ are $\pm\left(\xi_{1}^{2}+\xi_{2}^{2}\right)^{1 / 2}$.

\subsection{Lax-Friedrichs scheme}

We first consider the two-dimensional Lax-Friedrichs scheme (3). According to Theorem 1, the scheme is stable if (9) holds. In the particular case (25), (9) is equivalent to

$$
\forall \vartheta \in[0,2 \pi], \quad \lambda_{1}^{2} \cos ^{2} \vartheta+\lambda_{2}^{2} \sin ^{2} \vartheta \leq \frac{1}{2},
$$

and this condition is equivalent to $\max \left(\lambda_{1}, \lambda_{2}\right) \leq 1 / \sqrt{2}$.

Assume now that the scheme (3) is stable. Its symbol is given by (13). Using the notation $\zeta_{k}=\xi_{k} \Delta x_{k}, k=1,2$, we get

$$
\rho\left(G^{L F}\left(\xi_{1}, \xi_{2}\right)\right)^{2}=\frac{1}{4}\left(\cos \zeta_{1}+\cos \zeta_{2}\right)^{2}+\lambda_{1}^{2} \sin ^{2} \zeta_{1}+\lambda_{2}^{2} \sin ^{2} \zeta_{2}
$$

The spectral radius of $G^{L F}\left(\xi_{1}, \xi_{2}\right)$ is less than 1 for all $\left(\xi_{1}, \xi_{2}\right)$. Consequently, the mapping

$$
r: \quad\left(\zeta_{1}, \zeta_{2}\right) \longmapsto \frac{1}{4}\left(\cos \zeta_{1}+\cos \zeta_{2}\right)^{2}+\lambda_{1}^{2} \sin ^{2} \zeta_{1}+\lambda_{2}^{2} \sin ^{2} \zeta_{2},
$$

has a global maximum at the origin, therefore its hessian matrix at the origin is nonpositive. We compute

$$
D^{2} r(0,0)=\left(\begin{array}{cc}
2 \lambda_{1}^{2}-1 & 0 \\
0 & 2 \lambda_{2}^{2}-1
\end{array}\right)
$$

and we get $\max \left(\lambda_{1}, \lambda_{2}\right) \leq 1 / \sqrt{2}$.

\subsection{Godunov scheme}

When the matrices $A_{1,2}$ are given by (25), one computes $\left|A_{1}\right|=\left|A_{2}\right|=I_{2}$, and the symbol of the Godunov scheme (5) is given by

$$
\mathbb{G}=\left(1-2\left(\lambda_{1} \sin ^{2}\left(\frac{\xi_{1} \Delta x_{1}}{2}\right)+\lambda_{2} \sin ^{2}\left(\frac{\xi_{2} \Delta x_{2}}{2}\right)\right)\right) I_{2}-i\left(\lambda_{1} \sin \left(\xi_{1} \Delta x_{1}\right) A_{1}+\lambda_{2} \sin \left(\xi_{2} \Delta x_{2}\right) A_{2}\right) .
$$


In this case, the symbol $\mathbb{G}$ is normal for all $\left(\xi_{1}, \xi_{2}\right)$, and the scheme is stable if, and only if the spectral radius $\rho(\mathbb{G})$ does not exceed 1 for all $\left(\xi_{1}, \xi_{2}\right)$.

To simplify the subsequent calculations, we denote $\eta_{k}=\xi_{k} \Delta x_{k} / 2, k=1,2$. The eigenvalues of the symbol $\mathbb{G}$ are

$$
1-2\left(\lambda_{1} \sin ^{2} \eta_{1}+\lambda_{2} \sin ^{2} \eta_{2}\right) \pm i \sqrt{\lambda_{1}^{2} \sin ^{2}\left(2 \eta_{1}\right)+\lambda_{2}^{2} \sin ^{2}\left(2 \eta_{2}\right)} .
$$

After some simplifications, we thus compute

$$
\rho(\mathbb{G})^{2}=1+4\left(\lambda_{1}^{2}-\lambda_{1}\right) \sin ^{2} \eta_{1}+4\left(\lambda_{2}^{2}-\lambda_{2}\right) \sin ^{2} \eta_{2}+8 \lambda_{1} \lambda_{2} \sin ^{2} \eta_{1} \sin ^{2} \eta_{2} .
$$

The scheme (5) is thus stable if, and only if the following inequality holds true for all $\left(\eta_{1}, \eta_{2}\right) \in \mathbb{R}^{2}$ :

$$
\left(\lambda_{1}^{2}-\lambda_{1}\right) \sin ^{2} \eta_{1}+\left(\lambda_{2}^{2}-\lambda_{2}\right) \sin ^{2} \eta_{2}+2 \lambda_{1} \lambda_{2} \sin ^{2} \eta_{1} \sin ^{2} \eta_{2} \leq 0 .
$$

Choosing $\eta_{1}=\eta_{2}=\pi / 2$, (26) implies the necessary condition $\lambda_{1}+\lambda_{2} \leq 1$.

When $\lambda_{1}+\lambda_{2} \leq 1$, the scheme (5) is stable according to Theorem 1 . Therefore, the condition $\lambda_{1}+\lambda_{2} \leq 1$ is sufficient and necessary for the stability of (5).

\section{$5 \quad$ Numerical results}
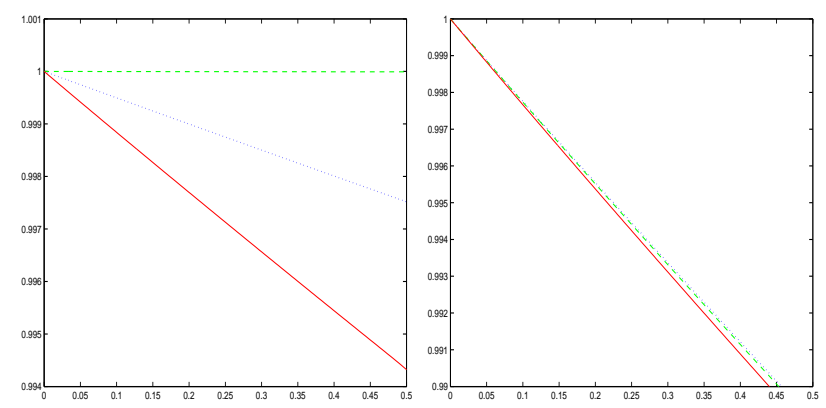

Figure 1: Left: scheme (3). Right: scheme (5)
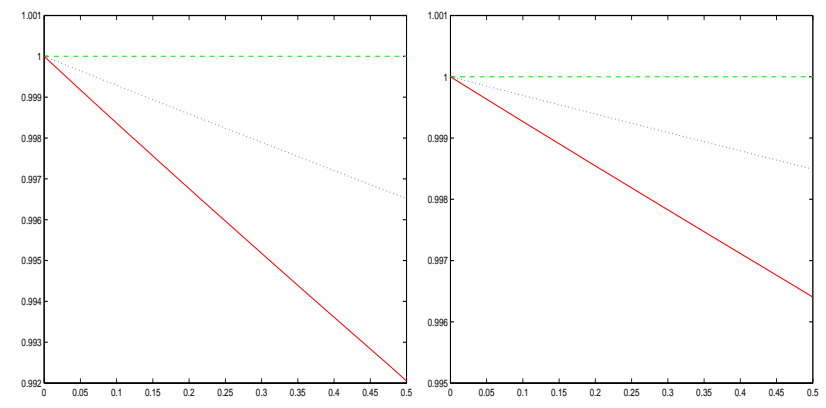

Figure 2: Left: schemes (4). Right: scheme (6).

In this section, we compare the dissipativity of the schemes $(3),(5),(4),(6)$. We consider the system

$$
\partial_{t} u+A_{1} \partial_{x_{1}} u+2 A_{2} \partial_{x_{2}} u=0,
$$

where the matrices $A_{1,2}$ are given by (25). (Observe the scaling in the $x_{2}$ variable). We run each of the four finite difference schemes on the square $[-2,2] \times[-2,2]$. To avoid the problem 
of boundary conditions, we choose some initial data that are supported in the square $[-1,1] \times$ $[-1,1]$, and we stop the computations when the support of the solution reaches the boundary (that is, at time $T=1 / 2$ ). The initial data are

$$
u_{0}\left(x_{1}, x_{2}\right)=\left(\begin{array}{c}
\left(\cos \left(x_{1} \pi / 2\right) \cos \left(x_{2} \pi / 2\right)\right)^{2} \\
\cos \left(x_{1} \pi / 2\right) \cos \left(x_{2} \pi / 2\right)
\end{array}\right)
$$

when $\left(x_{1}, x_{2}\right) \in[-1,1] \times[-1,1]$, and 0 outside.

In the $x_{2}$ direction, we consider a space step $\Delta x_{2}=4 / 300$ (which corresponds to 300 points), while in the $x_{1}$ direction, the space step is first $\Delta x_{1}=4 / 450$, then $\Delta x_{1}=4 / 600$, and, at last, $\Delta x_{1}=4 / 750$. We always choose the maximal time step that ensures stability (see Theorem 1 ). In the first case, one has $\lambda_{1} \rho\left(A_{1}\right)<\lambda_{2} \rho\left(2 A_{2}\right)$, in the second case, one has $\lambda_{1} \rho\left(A_{1}\right)=\lambda_{2} \rho\left(2 A_{2}\right)$, and in the last case, one has $\lambda_{1} \rho\left(A_{1}\right)>\lambda_{2} \rho\left(2 A_{2}\right)$. In figures 5, and 5, we plot the ratio $\|u(t)\|_{L^{2}} /\left\|u_{0}\right\|_{L^{2}}$ on the interval $[0, T]$. The dotted line represents the case $\Delta x_{1}=4 / 450$, the dashed line represents the case $\Delta x_{1}=4 / 600$, and the solid line represents the case $\Delta x_{1}=4 / 750$.

The numerical results show the following fact: the schemes (3), (4), and (6) do not diffuse when $\lambda_{1} \rho\left(A_{1}\right)=\lambda_{2} \rho\left(2 A_{2}\right)$, and in any case, (6) is the less diffusive scheme. Surprisingly, the two-dimensional Godunov scheme (5) has a more and more diffusive behavior as $\Delta x_{1}$ decreases. In particular, it is still diffusive when $\lambda_{1} \rho\left(A_{1}\right)=\lambda_{2} \rho\left(2 A_{2}\right)$.

These observations are easily explained by computing the modified equations of the finite difference schemes (3), and (5) (we shall not detail here the modified equations of the schemes $(4)$, and (6)). For the test case (27), the modified equation of the scheme (3) is

$$
\partial_{t} u+A_{1} \partial_{x_{1}} u+2 A_{2} \partial_{x_{2}} u=\Delta t\left[\left(\frac{1}{4 \lambda_{1}^{2}}-\frac{1}{2}\right) \partial_{x_{1} x_{1}}^{2} u+\left(\frac{1}{4 \lambda_{2}^{2}}-2\right) \partial_{x_{2} x_{2}}^{2} u\right] .
$$

In particular, when $\lambda_{1}=2 \lambda_{2}=1 / \sqrt{2}$, there is no diffusion in the modified equation.

For the test case (27), the modified equation of the scheme (5) is

$$
\partial_{t} u+A_{1} \partial_{x_{1}} u+2 A_{2} \partial_{x_{2}} u=\Delta t\left[\left(\frac{1}{2 \lambda_{1}}-\frac{1}{2}\right) \partial_{x_{1} x_{1}}^{2} u+\left(\frac{1}{\lambda_{2}}-2\right) \partial_{x_{2} x_{2}}^{2} u\right] .
$$

When $\lambda_{1}=2 \lambda_{2}=1 / 2$, there is a positive definite diffusion tensor in the modified equation, and the scheme (5) is dissipative. Note that the ideal choice would be $\lambda_{1}=2 \lambda_{2}=1$, but in this case the scheme is unstable according to Theorem 2 (this is confirmed by numerical simulations).

Acknowledgments Research of the author was supported by the EU financed network HYKE, HPRN-CT-2002-00282.

\section{References}

[1] E. Godlewski, P.-A. Raviart. Numerical approximation of hyperbolic systems of conservation laws, volume 118 of Applied Mathematical Sciences. Springer-Verlag, 1996.

[2] B. Gustafsson, H.-O. Kreiss, and J. Oliger. Time dependent problems and difference methods. Pure and Applied Mathematics. John Wiley \& Sons, 1995.

[3] A. Majda. Compressible fluid flow and systems of conservation laws in several space variables. Springer-Verlag, 1984.

[4] R. Vaillancourt. On the stability of Friedrichs' scheme and the modified Lax-Wendroff scheme. Math. Comp., 24:767-770, 1970. 